\title{
Confocal laser endomicroscopy for characterization of Crohn's disease-associated duodenitis
}

Confocal laser endomicroscopy (CLE) is a sophisticated endoscopic imaging technique in the armamentarium of modern endoscopy. By enabling real-time and in vivo visualization of cellular and subcellular details correlating with conventional histology, CLE has had tremendous impact on endoscopic diagnosis $[1,2]$. Several studies have described the utility of CLE for in vivo diagnosis of disorders of the upper gastrointestinal tract, such as Helicobacter pylori gastritis [3] and gastric metaplasia and cancer $[3,4]$. However, currently there are no available data regarding the utility of endomicroscopy for the in vivo diagnosis of Crohn's disease of the upper gastrointestinal tract.

Here, we report the case of a 41-year-old woman with ileocolonic Crohn's disease since 1995 . The patient was under antitumor necrosis factor (TNF) $\alpha$ therapy. She was initially admitted to our clinic as an inpatient reporting supraumbilical abdominal pain, especially in the evening and in the night, which required selfmedication with metamizole. We carried out esophagogastroduodenoscopy (EGD), which did not show any visible signs of inflammation in the stomach and duodenum ( $\bullet$ Fig.1a). After intravenous administration of $5 \mathrm{~mL} 10 \%$ fluorescein as the contrast agent, CLE (EC-3870 CIFK; Pentax, Tokyo, Japan) revealed dilated microvessels within the lamina propria of the duodenum and mild leakage of fluo- rescein sodium. In addition, some regenerative crypt changes were also seen ( Fig. 1b). The endomicroscopic diagnosis of moderate mucosal inflammation was confirmed on corresponding histopathological examination ( $\bullet$ Fig. 1 c). In summary, based on the above findings, using fluorescein-guided endomicroscopy we detected in vivo and in real time, a duodenal manifestation of the patient's Crohn's disease. Here, we highlight the clinical usefulness of CLE in the diagnosis of duodenal involvement of Crohn's disease. The new duodenal involvement was considered indicative of disease progression and therefore we decided to intensify the current anti-TNF $\alpha$ therapy. Prospective, blinded imaging studies are required to evaluate the accuracy, sensitivity, and specificity of this endopathological approach.

Endoscopy_UCTN_Code_CCL_1AB_2AZ_3AC

Competing interests: None

\section{G. Hundorfean', A. Agaimy², R. Atreya', J. Mudter ${ }^{1}$, M. F. Neurath ${ }^{1}$, H. Neu- mann ${ }^{1}$}

${ }^{1}$ Medical Clinic I, University of ErlangenNuremberg, Erlangen, Germany

${ }^{2}$ Institute of Pathology, University of Erlangen-Nuremberg, Erlangen, Germany

\section{References}

1 Neumann H, Kiesslich $R$, Wallace MB et al. Confocal laser endomicroscopy: technical advances and clinical applications. Gastroenterology 2010; 139 (2): 388-392, 92 e1-2

2 Inoue H, Kudo SE, Shiokawa A. Technology insight: Laser-scanning confocal microscopy and endocytoscopy for cellular observation of the gastrointestinal tract. Nat Clin Pract Gastroenterol Hepatol 2005; 2 (1): 31-37

3 Kiesslich R, Goetz M, Burg J et al. Diagnosing Helicobacter pylori in vivo by confocal laser endoscopy. Gastroenterology 2005; 128 (7): 2119-2123

4 Guo YT, Li YQ Yu T et al. Diagnosis of gastric intestinal metaplasia with confocal laser endomicroscopy in vivo: a prospective study. Endoscopy 2008; 40 (7): 547-553

\section{Bibliography}

Dol http://dx.doi.org/

10.1055/s-0031-1291605

Endoscopy 2012; 44: E80

Stuttgart - New York

ISSN 0013-726X

\section{Corresponding author}

\section{H. Neumann (Professor of Medicine)}

Medical Clinic I

University of Erlangen-Nuremberg

Erlangen

Germany

helmut.neumann@uk-erlangen.de
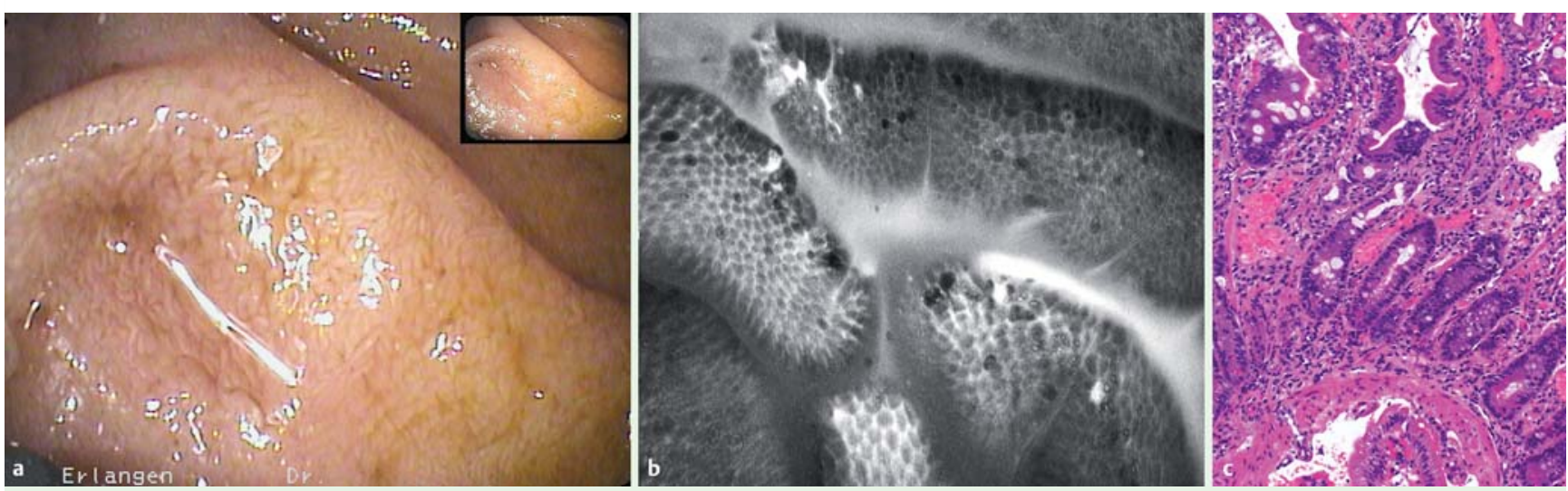

Fig. 1 a High-resolution endoscopy in a 41-year-old woman with ileocolonic Crohn's disease showing a macroscopically normal duodenal mucosa. b Confocal laser endomicroscopy showing dilated microvessels within the lamina propria, mild fluorescein sodium leakage, and some regenerative changes. $\mathrm{c}$ A histopathological section from the duodenal biopsy specimen taken from the same area shows hemorrhagic inflammation and epithelial regeneration (hematoxylineosin stain), confirming our endomicroscopic findings. 